Full length article

\title{
A hybrid method for reconstruction of three-dimensional heterogeneous porous media from two-dimensional images
}

\author{
Lili $\mathrm{Ji}^{\mathrm{a}}$, Mian Lin ${ }^{\mathrm{a}, \mathrm{b}, *}$, Wenbin Jiang ${ }^{\mathrm{a}}$, Gaohui Cao ${ }^{\mathrm{a}, \mathrm{b}}$ \\ ${ }^{\text {a }}$ Key Laboratory for Mechanics in Fluid Solid Coupling Systems, Institute of Mechanics, Chinese Academy of Sciences, Beijing 100190, China \\ ${ }^{\mathrm{b}}$ School of Engineering Science, University of Chinese Academy of Sciences, Beijing 100190, China
}

\section{A R T I C L E I N F O}

\section{Keywords:}

Heterogeneous porous media

Reconstruction

The hybrid method

Tight sandstones

Pore-size distribution

\begin{abstract}
A B S T R A C T
The heterogeneous pore space of porous media strongly affects the storage and migration of oil and gas in the reservoir. To accurately reproduce complex pore structure validated with experiment data, a hybrid method which combines the CCSIM-TSS method (the cross correlation based simulation-three step sampling method) with the optimization method is proposed to reconstruct stochastically 3D models of the heterogeneous porous media based on 2D images and some constrain conditions. Moreover, the new system in the hybrid method is generated by exchanging the objective points and boundary points of pore and matrix based on two algorithms. Then the hybrid method are tested on the two typical samples (carbonate rock, tight sandstone samples with micro-nanopores). Quantitative comparison is made by computing various statistical and petrophysical properties for the reconstructed models, as well as the original samples. It was found that the hybrid method generates an ensemble of stochastic realization that honor the constrain conditions (permeability data, pore-size distributions and the connectivity). Generating realization of tight sandstones that match the experimental data based on 2D SEM image shows that this method may be then used for more accurate characterization of tight sandstones oil reservoir and analysis of their pore network.
\end{abstract}

\section{Introduction}

Complex pore structures have considerable influence on the storage and migration of oil and gas in the reservoir, thus constructing digital core and characterization of pore structures play a vital role in the exploration of reservoirs (Weger et al., 2009; Payne et al., 2010). The heterogeneous porous media, such as tight sandstones and carbonate rocks, are highly heterogeneous and many of them contain a complex pore structure with a wide range of length scales. Many methods for modeling of porous media cannot be used for these heterogeneous porous media. Moreover, most of the current modeling methods may not be able to fully reproduce the experimental data for the permeability and pore size distribution. Hence, it is of great significance to develop methods to accurately describe the pore structure and permeability of heterogeneous porous media.

Digital core construction methods are in general divided the following two classes: (1) the physical construction method, focused ion beam scanning electron microscopy (FIB-SEM) or X-ray Computed Tomography (CT), can directly construct real 3D digital cores (Bai et al., 2013; Chen and Zhou, 2017; Curtis et al., 2012; Kelly et al., 2016; Liu et al., 2017; Zhou et al., 2016). Micro-CT can only resolve meso pores and part of micro pores and can't identify extra small $(\mathrm{nm})$ pores in tight sandstones. Although nano-CT and FIB-SEM can identify the nano-scale pores, the field of view of them is too small to reflect bulk properties. Also, the high cost, computational difficulty, and the timeintensive effort required, providing 3D datasets for different samples has become a challenging problem. On the other hand, 2D images can be obtained with ease at a larger scale (hundreds of $\mu \mathrm{m}$ ) and at a lower cost and much higher efficiency. (2) Computer technology and mathematical modeling methods can reconstruct 3D structures from a single high-resolution two-dimensional (2D) image. Such models may be divided into three groups: process-based methods, object-based techniques and pixel-based methodologies. Process-based methods (Bryant and Blunt, 1992; Biswal et al., 1999, 2007; Coelho et al., 1997; Øren and Bakke 2002) try to develop 3D models by mimicking the physical processes that form the porous medium. Though realistic, such methods are, however, computationally expensive and require considerable calibrations. Moreover, they are not general enough, because each of them is developed for a specific type of rock, as each type is the outcome of some specific physical processes. In the object-based techniques, the pore and grain structures are treated as a set of objects that are defined based on the prior knowledge of the pore space (Pyrcz and

\footnotetext{
* Corresponding author at: No. 15 Beisihuanxi Road, Beijing 100190, China

E-mail addresses: linmian@imech.ac.cn, jiangwenbin@imech.ac.cn (M. Lin).
} 


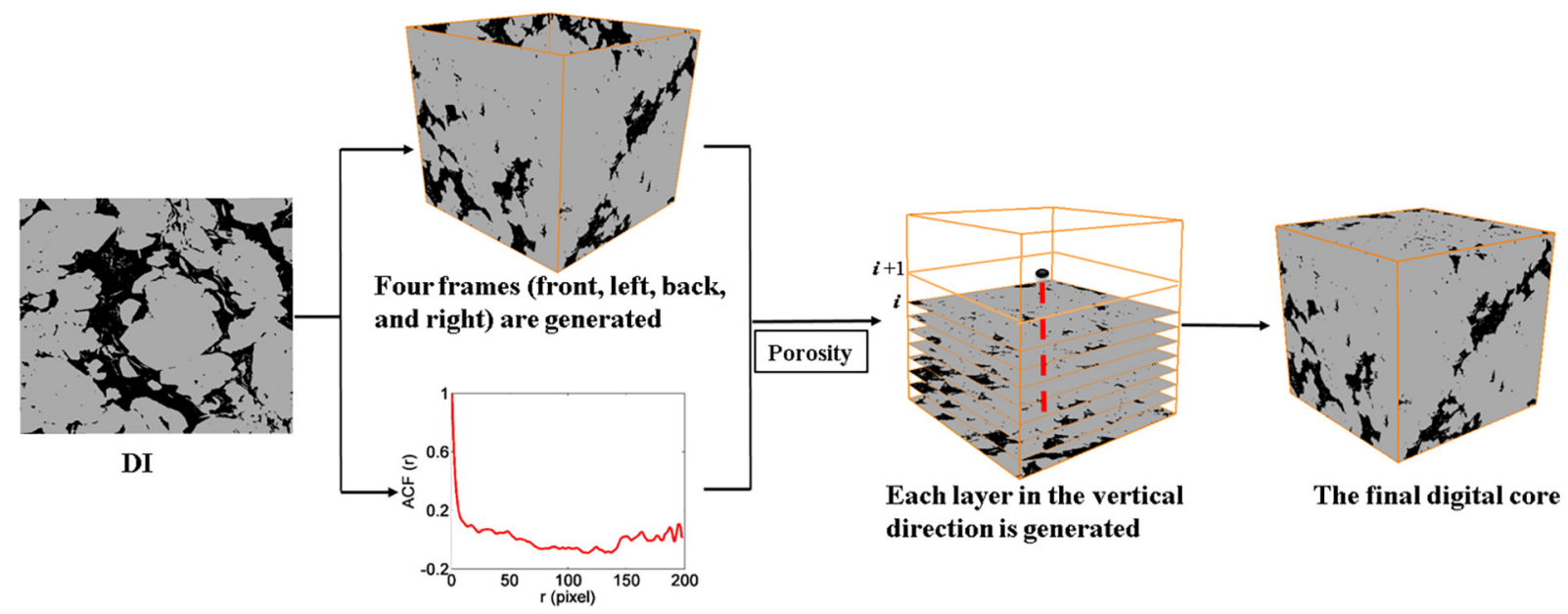

Fig. 1. The schematic illustration of the CCDIM-TSS algorithm.

Deutsch, 2014). Simulated annealing, hierarchical simulated annealing (HSA), and Gaussian random field, are the most typical algorithms of this classification. Stability is the most significant advantage of this kind of algorithms, which ensures that the reconstructed results will have the characteristics specified by the objective function when they converge to it. However, the object-based techniques fail to reproduce the longrange connectivity of the pore space, especially for low porosity materials and particulate media with special shapes, because only the loworder information is used. Pixel-based methodologies are based on defining an array of points or pixels in a regular grid, with the pixels representing various properties of a reservoir. Unlike the object-based techniques, they can be used effectively to produce the shapes that are hard to fit to a specific regular object. However, these techniques are unable to produce very realistic pore structures. Single normal equation simulation method (SNESIM), the Markov stochastic reconstruction method (MCMC) (Wu et al., 2004), the cross correlation based simulation (CCSIM) and their derivative algorithms belong to this group. The single normal equation simulation (SNESIM) proposed by Strebelle (Strebelle, 2002; Strebelle and Cavelius, 2014) uses a search tree structure, an efficient method of accessing high-dimensional data that can overcome the problems associated with the original MPS method. The method is computationally demanding and unable to control the continuity and variability among adjacent layers (Hajizadeh et al., 2011; Hajizadeh and Farhadpour, 2012). Further, they have never been tested for reconstructing models of shale reservoirs. The cross-correlation-based simulation (CCSIM) method, proposed by Tahmasebi et al. (2012, 2015, 2016), was used to reconstruct stochastically equiprobable 3D models of shale rocks. This method produces an ensemble of 3D realizations that provide acceptable approximation of the same properties in the 2D image(s). However, the reconstructed vertical morphological features are unsatisfactory (Gao et al., 2015; Tahmasebi and Sahimi, 2016), and also the method is unable to reproduce accurately the experimental data for the permeability and electrical conductivity. The CCSIM-TSS method (the cross correlation based simulation-three step sampling method) is derived from the cross correlation based simulation (CCSIM) to improve the long-range (global) connectivity of the pore space in the vertical direction (Ji et al., 2018). However, as other pixel-based methodologies, it is not capable of reproducing accurately the experimental data for the permeability and pore size distribution. Thus in this paper, to reproduce more realistic morphology for porous media, we develop a hybrid method which combines the CCSIM-TSS method with the multiple objective simulated annealing algorithm to generate 3D digital cores based on 2D images. In other words, the strength of pixel-based method for producing complex pore space and object-based methods for realistic models are integrated.

The rest of this paper is organized as follows. In the next section we describe the hybrid method that we propose in this paper, including an algorithm (CCSIM-TSS method) that was recently suggested and utilized in the present paper, and how it is integrated with the optimization method based on multiple objective simulated annealing algorithm (MOSA). Then the accuracy of the new algorithms is tested using carbonate rocks and tight sandstones. Finally the paper concludes with a summary and discussion of the results.

\section{Methodology}

In this paper the CCSIM-TSS algorithm (the cross correlation based simulation-three step sampling method) is integrated with an optimization method scheme based on the multiple objective simulated annealing method to generate an ensemble of 3D realizations that reproduce the experimental results. The hybrid method starts with the CCSIM-TSS algorithm to build a 3D digital core which is the initial configuration for the optimization method based on the MOSA method. The 3D digital core is adjusted by the optimization method to minimize the difference between the reconstructed and realistic models. In particular, the two-point connectivity probability functions, the pore-size distribution functions and the permeability are used as the constraint conditions in the optimization method.

\subsection{Ccsim-TSS}

In the beginning of the hybrid method proposed in this paper, the CCSIM-TSS algorithm is used to generate an ensemble 3D realization. The CCSIM-TSS algorithm is a newly developed algorithm which combines the cross correlation based simulation (CCSIM) with the three step sampling method together to reconstruct stochastically 3D models of the heterogeneous porous media ( $\mathrm{Ji}$ et al., 2018). It can provide acceptable approximation of the same properties in the $2 \mathrm{D}$ image(s) and preserve the long-range (global) connectivity of the pore space in the vertical direction. However, it produces a range of realizations instead of one single value and is unable to produce very realistic pore structures.

The specific process of CCSIM-TSS is arranged as follows (as show in Fig. 1):

(1) At first, typical 2D digital images (DIs), which include most of the expected variations and heterogeneity of the porous medium, are selected.

(2) The original DI is set as the first layer at the bottom of 3D model. Then, the other four frames (i.e., front, left, back, and right) are generated using the cross correlation based simulation (CCSIM), as shown in Fig. 1. 
(3) Now we reconstruct each layer of the digital core in the vertical direction. It should be pointed out that the two-point connectivity probability function (ACF (r)) is calculated based on the typical digital images (DIs). Let $i+1$ be the number of layers to be reconstructed currently. We select the condition data as follows: (a) The ith layer is scanned using a $5 \times 5$ sampling template, and the central node of the template, which is entirely pore (grain), is marked as a sampling point. The number of sampling points is recorded, and this sampled area is labeled. (b) The remaining portion of the image is scanned using a $3 \times 3$ sampling template, and the sampling points are selected. (c) The unavailable sampling area is labeled, and the number of pixels is recorded. (d) The edge area is marked and the sampling points in the edge area are selected based on the two-point connectivity probability function. The procedure continues until all the (2D) layers in the vertical have been reconstructed.

(4) Eventually, all the generated layers are stacked together to create the reconstructed 3D model.

\subsection{The optimization method}

The optimization method based on multiple objective simulated annealing algorithm (MOSA) can be seen as an optimization problem to minimize the difference between the properties of the reconstructed 3D digital core and the realistic one. We design the subsequent search scheme, defines the criterion to select the boundary point of pore and the matrix, and proposes the generation solutions of the new system by exchanging the isolated pore points and boundary points of pore and matrix based on two algorithms. The objective functions used in this paper is the two-point connectivity probability functions, pore-size distribution functions and the permeability obtained through experiments.

The flow chart of the optimization method is shown in Fig. 2. In the following, we describe its' components in a detailed manner:

(1) The digital core reconstructed by CCSIM-TSS is used as the initial configuration for the optimization method and the initial temperature $T_{0}$ are assumed. Then the three objective functions of the initial system is calculated, and the objective functions of the system is defined as follows:

$$
\begin{gathered}
f_{1}=\sum_{i} \alpha_{i}\left[S_{x i}^{r}-S_{x i}\right]^{2}+\sum_{i} \beta_{i}\left[S_{y i}^{r}-S_{y i}\right]^{2}+\sum_{i} \gamma_{i}\left[S_{z i}^{r}-S_{z i}\right]^{2} \\
f_{2}=\sum_{i} \kappa_{i}\left[P_{i}^{r}-P_{i}\right]^{2} \\
f_{3}=\left[\eta_{x}\left(K_{x}^{r}-K_{x}\right)^{2}+\eta_{y}\left(K_{y}^{r}-K_{y}\right)^{2}+\eta_{z}\left(K_{z}^{r}-K_{z}\right)^{2}\right]
\end{gathered}
$$

where $\alpha_{i}, \beta_{i}, \gamma_{i}, \kappa_{i}$ is the weight for the $i$-th statistical property. $S_{x i}^{r}, S_{y i}^{r}, S_{z i}^{r}$, $S_{x i}, S_{y i}, S_{z i}$ are the two- point connectivity probability functions in $\mathrm{X}, \mathrm{Y}$ and $\mathrm{Z}$ directions of DIs and the models. $P_{i}^{r}, P_{i}$ are the pore-size distribution functions obtained from experiments and the models, $K_{x}^{r}, K_{x}, K_{y}^{r}, K_{y}, K_{z}^{r}, K_{z}$ are the permeability obtained from experiments and the models, and $\eta_{x}, \eta_{y}, \eta_{z}$ are the weight for the permeability in three directions.

The AB (axis \& ball) algorithm (Yi et al., 2017), a newly developed pore network extraction algorithm, is used to extract the pore networks from the digital cores. Then the pore-size distribution is calculated.

(2) The three objective functions of the current system are evaluated and the maximum objective function are chosen: $f=\max \left(f_{1}, f_{2}, f_{3}\right)$.

(3) The iteration number for the current temperature are given, $n\left(T_{k}\right)$

(4) New configurations are generated by exchanging the objective points and boundary points of pore and matrix based on two algorithms.

The efficient generation of new configurations requires the location of two voxels at the two-phase interface, and their subsequent exchange. We further refined this method of voxel location by the implementation of two algorithms:

Algorithm I. if $f=f_{1}$ or $f_{3}$, then the connectivity of the current configuration should be improved based on Algorithm I. In this algorithm, the D3Q19 model is used to select the object point. As shown in Fig. 3a, the voxel in the center of the D3Q19 lattices structure model is denoted as 0 . If there are $n$ voxels of the 19 voxels in the neighborhood of the voxel 0 in the different phase with the voxel 0 , the voxel 0 is selected as the object point. It should be pointed out that the value of $\mathrm{n}$ is determined according to the specific circumstances of the case. If $\mathrm{n}$ is too large, the object voxel will become difficult to be found, and it will take much time to find the object voxel. However, if $\mathrm{n}$ is too small, the connectivity of the current configuration will be destroyed. In this paper, $n$ is 5 as the voxel 0 is pore, while $n$ is 11 as the voxel 0 is matrix. In particular, the voxel 0 is defined as complete pore when the voxel 0 and the 19 voxels in its neighborhood are all pores. Let the coordinate of the complete pore 0 is $(i, j, k)$. Next we select the boundary point and exchange it with the object voxel to generate the new configuration. As shown in Fig. 3b, A and B denote the positive and negative direction of $\mathrm{X}$-axis, while $\mathrm{C}(\mathrm{E})$ and $\mathrm{D}(\mathrm{F})$ denote the positive and negative direction of $\mathrm{Y}$-axis (Z-axis). One of the above six directions is chosen randomly. Then we start from the complete pore $0(\mathrm{i}, \mathrm{j}, \mathrm{k})$ and search the boundary voxel along the above chose direction. It should be pointed out that if the boundary voxel is not found along this direction, and then we should choose another direction to find the boundary voxel. A new trial state is obtained by interchanging the object voxel and the boundary voxel. At last, it should be emphasized that if the difference of the two-point connectivity probability function in one direction, such as in y direction, is bigger than the other two directions, we should choose boundary voxels along y directions.

Algorithm II. if $f=f_{2}$, then the pore network of the current configuration should be improved based on Algorithm II. Firstly, the $\mathrm{AB}$ algorithm is used to extract the pore networks from the current configuration, and the pore-size distribution p'(r) is calculated. Next, $\mathrm{p}^{\prime}(\mathrm{r})$ is compared with the pore-size distribution $\mathrm{p}(\mathrm{r})$ of experiments. Based on the results, the pores are classified into three types: $U_{1}\left(r_{1}, r_{2}\right.$, $\left.\ldots, r_{n}\right), U_{2}\left(r_{1}^{0}, r_{2}^{0}, \ldots, r_{n}^{0}\right)$ and $U_{3}\left(r_{1}^{1}, r_{2}^{1}, \ldots, r_{n}^{1}\right)$. Any point $r_{i}$ in the set $U_{1}$ denotes the points that the number of points with the radius $r_{i}$ in the current configuration is smaller than that in the real rock sample. Any point $r_{i}^{0}$ in the set $U_{2}$ denotes the points that the number of points with the radius $r_{i}$ in the current configuration is bigger than that in the real rock sample. Any point $r_{i}^{1}$ in the set $U_{3}$ denotes the points that the number of points with the radius $r_{i}$ in the current configuration is equal to that in the real rock sample. Two pores are chosen from $U_{1}$ and $U_{2}$ at random, respectively. The centers of the two pores are denoted by 01 and 02, and obviously, 01 and 02 are complete pores. Beginning at the starting point 01 , the boundary voxels of 01 are sought in six different directions, as shown in Fig. 3b. In the same way, the boundary voxels of 02 are sought and then exchanged with the six boundary voxels of 01 . Finally, we obtain the new trial state.

\section{(5) The objective functions of the trial configuration is recalculated.}

For $f=f_{1}$ or $f_{2}$, if $f\left(X_{n}\right)>f\left(X_{n+1}\right)$ the trial configuration is unconditionally accepted and becomes the current configuration. On the other hand, if $f\left(X_{n}\right)<f\left(X_{n+1}\right)$, then the trial configuration is accepted with a probability given by

$p(\Delta f)=\exp \left(\frac{f\left(X_{n}\right)-f\left(X_{n+1}\right)}{T_{k}}\right)$

where $\Delta \mathrm{f}=f\left(X_{n}\right)-f\left(X_{n+1}\right)$.

For case $f=f_{3}$, it is important to point out that in this step (the iteration for the current temperature) the objective function is not the permeability. The multi-point connectivity functions (Krishnan and 


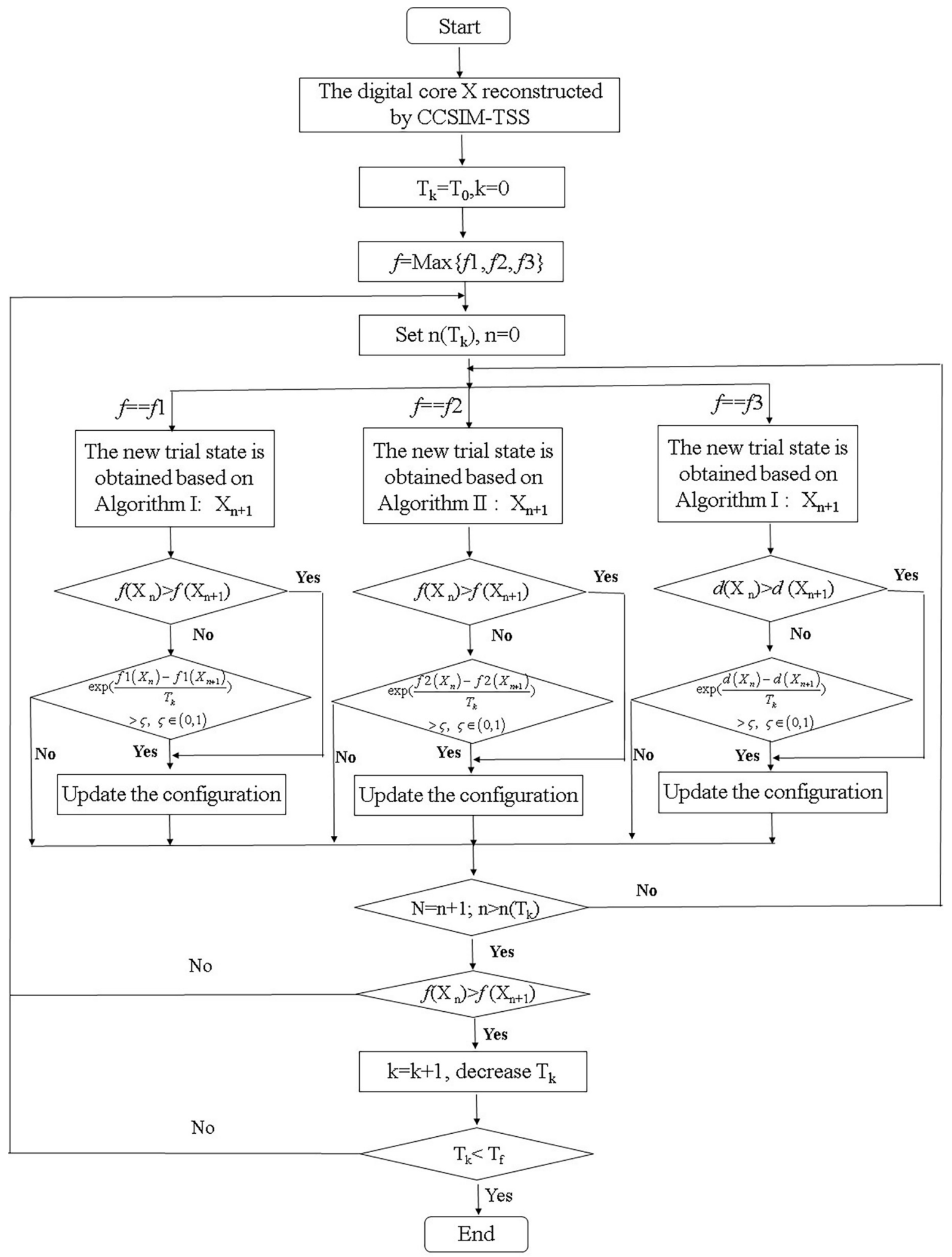

Fig. 2. The flow chart of the optimization method.

Journel, 2003) are used here to evaluate the quality of the trial configuration. This is because that the trial configuration is obtained by exchange only two voxels and its permeability can't change obviously compared to the old configuration. However, the multi-point connectivity functions of the trial configuration have obvious difference with that of the old configuration. Moreover, the multi-point connectivity functions allows one to account for the curvilinearity of the system by considering a tolerance core around a target direction, and it can reflect the permeability of the digital core. Thus here we use the difference between the curve of the multiple-point connectivity probability function curve and the straight line between the beginning and the end of this curve (defined as the reference line) as the objective function. For example, let the multiple-point connectivity probability function in three directions is shown in Fig. 4, and then $d x, d y$ and $d z$ are 


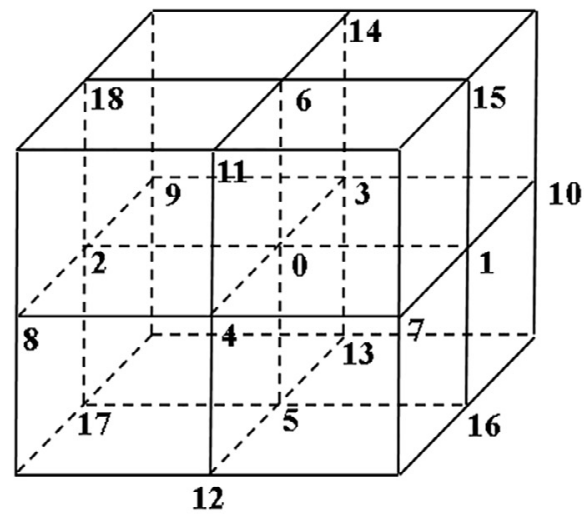

(a)

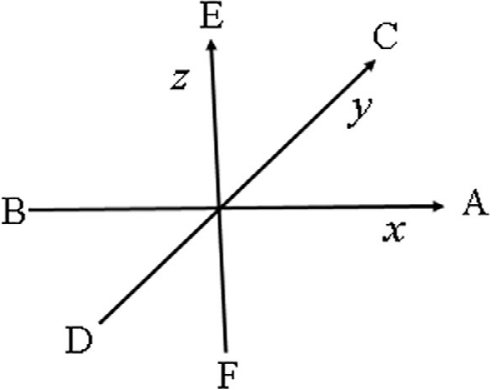

(b)

Fig. 3. The D3Q19 lattices structure model (a) and the diagram of six directions (b).

calculated as follows:

$d_{\eta}=\sum_{i=1}^{n}\left|p_{\eta}\left(r_{i}\right)-p_{r}\left(r_{i}\right)\right|$

$$
(\eta=x, y, z)
$$

where $d_{\eta}(\eta=x, y, z)$ is the absolute value of the difference between the curve of the multiple-point connectivity probability function along $\mathrm{X}, \mathrm{Y}, \mathrm{Z}$ direction and the reference line. $p_{\eta}\left(r_{i}\right)$ is the multiple-point connectivity probability function along $\mathrm{X}, \mathrm{Y}, \mathrm{Z}$ direction. $p_{r}\left(r_{i}\right)$ is the reference line which is defined as the straight line between the beginning and the end of the multiple-point connectivity probability function curve (Fig. 4), and $\mathrm{n}$ is the number of pixels in the figure.

As $d_{\eta}(\eta=x, y, z)$ decreases, the connectivity of pore structure in corresponding direction is becoming better and the corresponding permeability increases. Therefore, we can adjust the value of $d_{\eta}$ $(\eta=x, y, z)$ to minimize the difference of permeability between the reconstructed model and the real rock sample.

(6) Repeat Steps 2-5 until a predefined number of iterations $n\left(T_{k}\right)$ is carried out.

(7) The three objective functions $f_{1}, f_{2}, f_{3}$ (the permeability) are reevaluated. If $f\left(X_{n}\right)<f\left(X_{n+1}\right)$, then reduce the temperature using a problem dependent annealing schedule, and go to Step 2. On the other hand, if $f\left(X_{n}\right)>f\left(X_{n+1}\right)$, go to Step 2 directly.

(8) This process (2)-(7) is repeated until $T_{k}$ becomes lower than a specified value.

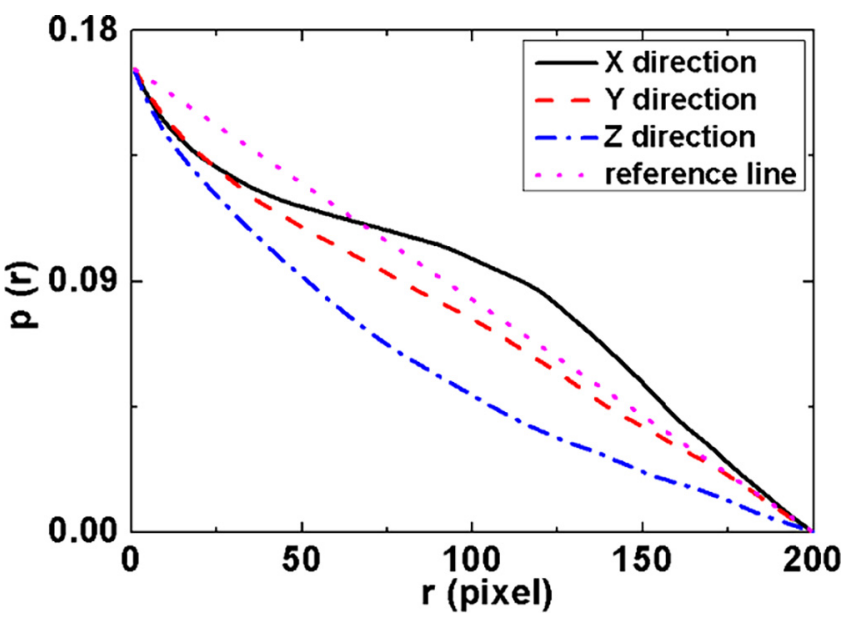

Fig. 4. Curves of the multiple-point connectivity probability functions and the reference line of the rock sample.
It's should be noted that in order to avoid destroying the connectivity of the digital core, we only randomly select pairs of voxels on the pore-matrix interface, one pore voxel and one matrix voxel, to interchange their phases. As the number of voxels that have changed their phases successfully increases, the auto-correlation function, the poresize distribution and the permeability of the 3-D digital core converges gradually to that of the actual samples.

In the following we will use three different rock samples (carbonate rock, tight sandstone sample and sandstones) to test the described methodology.

\section{Case study and parameter analysis}

\subsection{Carbonate rock}

To investigate the reconstruction of heterogeneous porous media, we select a carbonate sample, referred to as $\mathrm{C}$, for reconstruction. The carbonate rock 3D Micro-CT-scan data consist of 200 2D slices with $200 \times 200$ elements and a voxel size of $10.69 \mu \mathrm{m}$. The porosity of the carbonate sample is $16.8 \%$. The absolute permeability of the carbonate rock calculated through $\mathrm{AB}$ (axis \& ball) algorithm (Yi et al., 2017) based on the Micro-CT image are $198 \mathrm{mD}, 323 \mathrm{mD}$ and $248 \mathrm{mD}$ in three directions.

Three layers in the three directions among the Micro-CT image as representative images of the carbonate sample, referred to as DIx, DIy and DIz, is selected to reconstruct the 3D model of sample C ( $\mathrm{Ji}$ et al., 2018). Then DIx is used as the bottom of the 3D model, as shown in Fig. 5a. Also, based on the three representative images, the two-point connectivity probability function (ACF (r)) are extracted (Fig. 5b), and

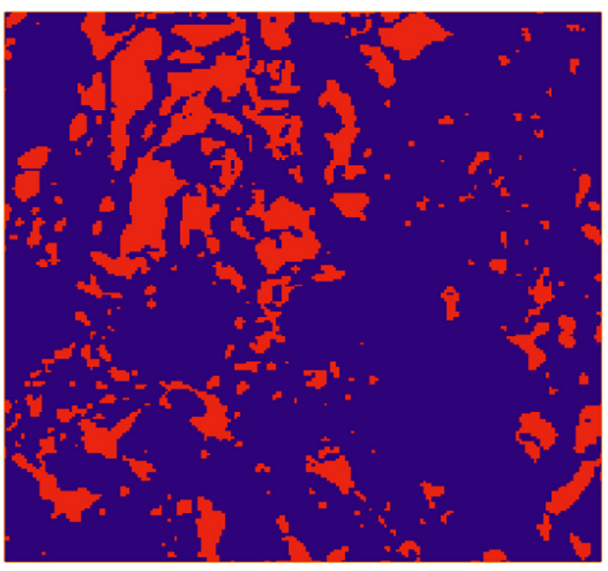

Fig. 5a. The extracted 2D representative image in the $\mathrm{x}$ direction. 


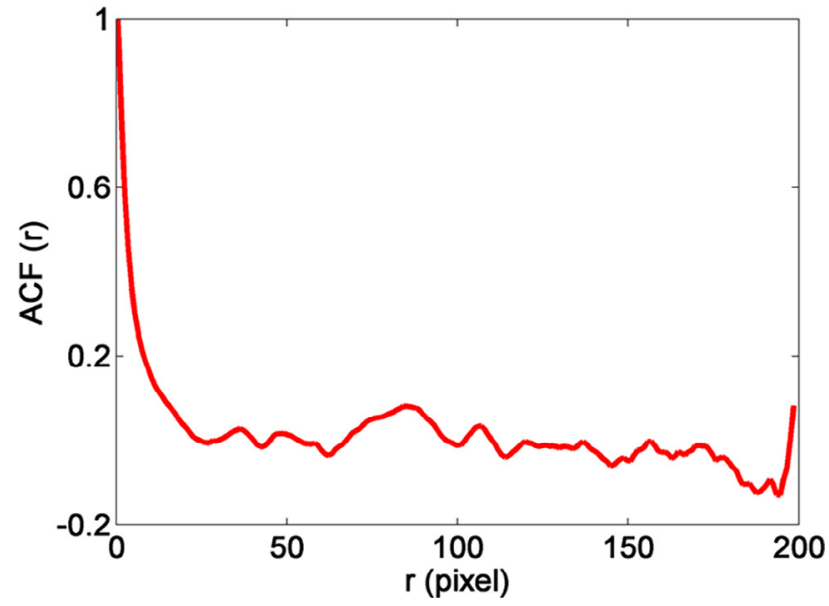

Fig. 5b. The curve of the two-point connectivity probability function extracted as the vertical constrain condition.

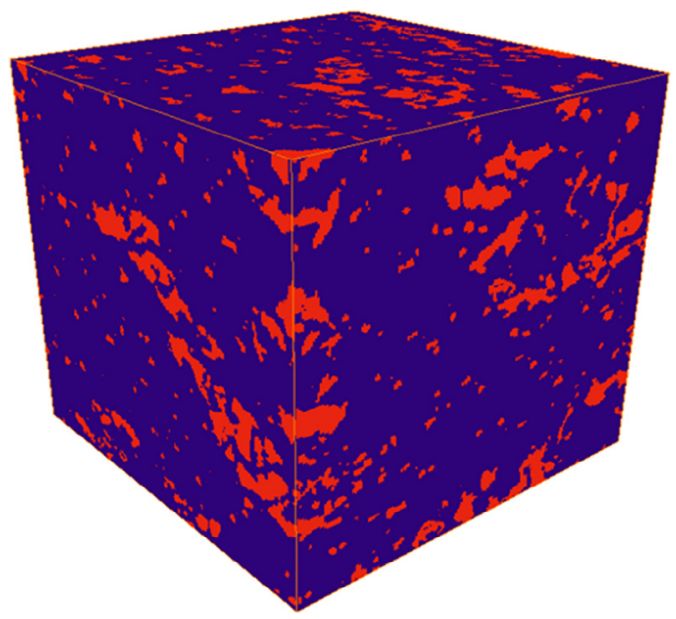

Fig. 5c. The reconstructed model by CCSIM-TSS method.

it will be used as vertical constraint conditions in CCSIM-TSS.

Fig. $5 \mathrm{c}$ plots the realization generated by CCSIM-TSS, and then the connectivity and the pore-size distribution of the produced realization are estimated. First, the difference of the two-point connectivity probability functions between the realization and the Micro-CT image, was compared, as shown in Fig. 6. The plots indicate that there is a difference of connectivity in three directions. Further, the pore-size distribution of the realizations for carbonate sample are calculated, and then they are compared with the pore-size distribution of the Micro-CT image. It can be seen from Fig. 7 that the pore-size distribution of Micro-CT image and the realization is relatively big. Finally, the

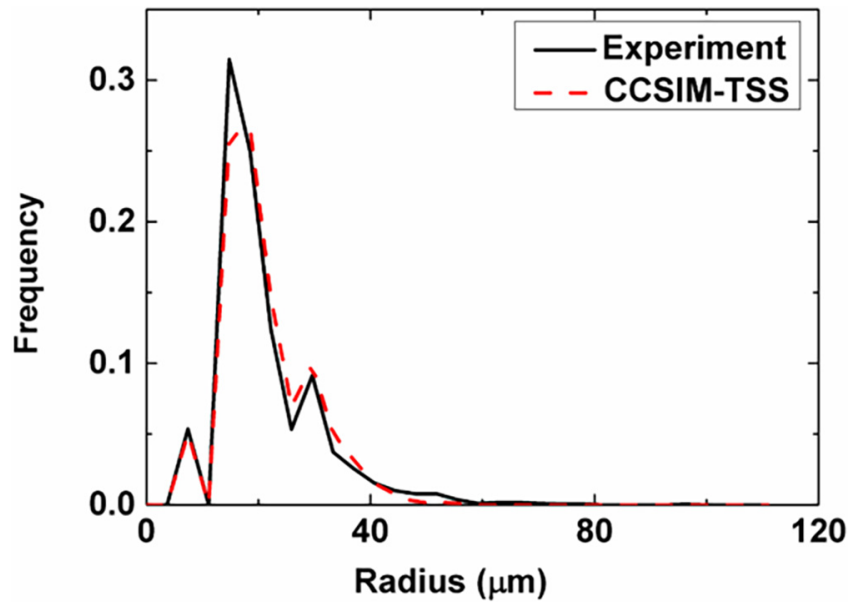

Fig. 7. Curves of the pore-size distribution of the reconstructed results (red) and experiment (black) for carbonate sample C. (For interpretation of the references to colour in this figure legend, the reader is referred to the web version of this article.)

absolute permeability of the realizations for carbonate sample are evaluated, and they are $140 \mathrm{mD}, 201 \mathrm{mD}$ and $117 \mathrm{mD}$. Therefore, it can be obtained that the realizations generated by the CCSIM-TSS method is not capable of reproducing accurately the connectivity and the poresize distribution of the actual sample. Thus in the following we will use the optimization method to adjust the realization to minimize the difference between the realization and the actual sample.

To minimize the difference of the reconstructed model and the real rock sample, the second step of the hybrid method, the optimization method, is used to optimize the reconstructed model. Inspecting Fig. 6, we can find that when $r \geqslant 20$ the difference of the two-point connectivity probability is relatively big. Thus $\alpha, \beta, \gamma$ for $r \geqslant 20$ is assumed to be twice as big as that for $r<20$. Similarly, $x$ for $10 \mu \mathrm{m} \leqslant r \leqslant 30 \mu \mathrm{m}$ is assumed to be twice as big as that for $r<10 \mu \mathrm{m}$ and $r>30 \mu \mathrm{m}$. It should be pointed out that the permeability in three direction of the reconstructed model is smaller than that of the real rock sample. Thus during iterative process, the $d_{\eta}(\eta=x, y, z)$ should be reduced to improve the connectivity of the pore structure in three directions.

After 238 iterations, we obtain the final reconstructed result by the optimization method. Then the connectivity in three directions and the pore-size distribution of the produced realization are estimated. As shown in Fig. 8 the two-point connectivity function in three directions of the final realization is much closer to that of the Micro-CT image than CCSIM-TSS result. Also, the pore-size distribution of the final reconstructed result and the experiment for sample $\mathrm{C}$ is compared in Fig. 9. The figure indicates that the final realization reproduces the pore-throat size distribution of the original sample. Thus we can obtain that the final result obtained by the optimization method can reflect the
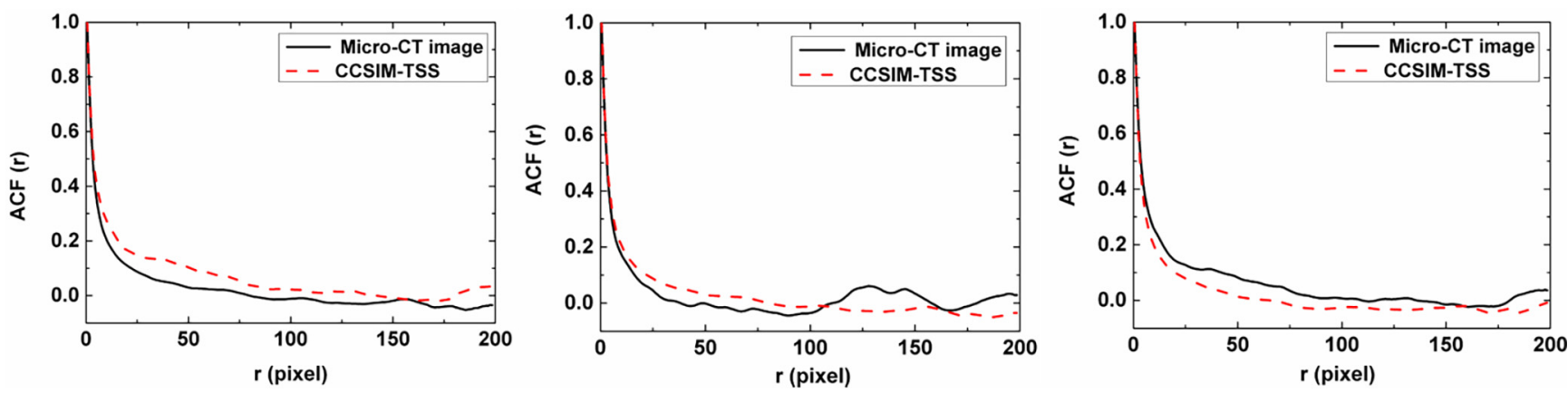

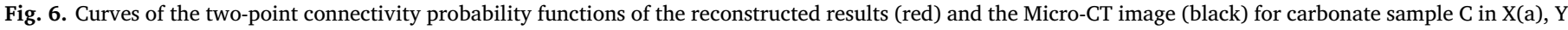
(b) and Z(c) direction. (For interpretation of the references to colour in this figure legend, the reader is referred to the web version of this article.) 

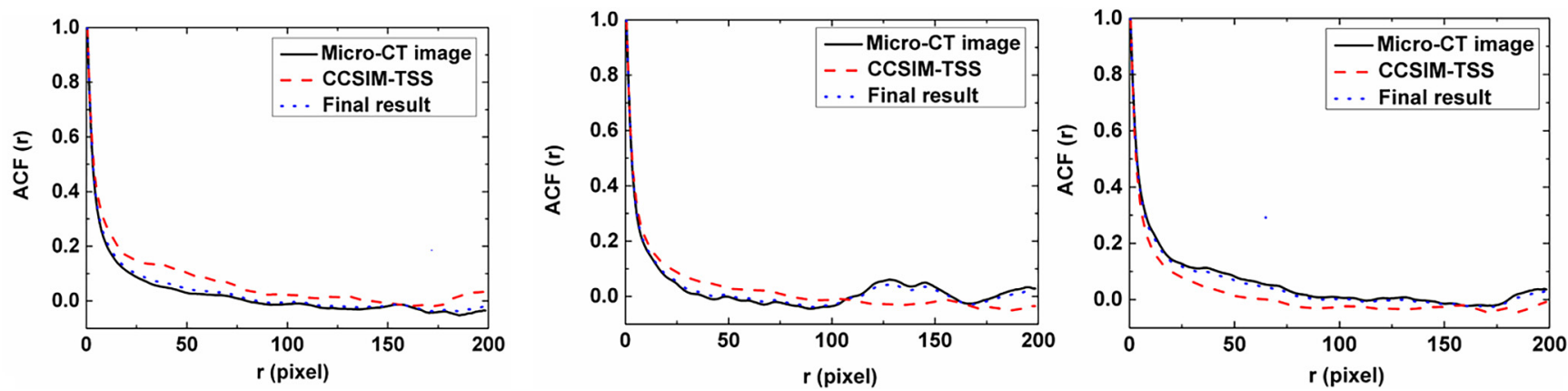

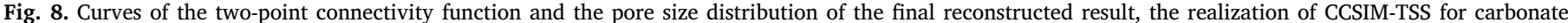
sample $\mathrm{C}$ in $\mathrm{X}(\mathrm{a}), \mathrm{Y}(\mathrm{b})$ and $\mathrm{Z}(\mathrm{c})$ direction.

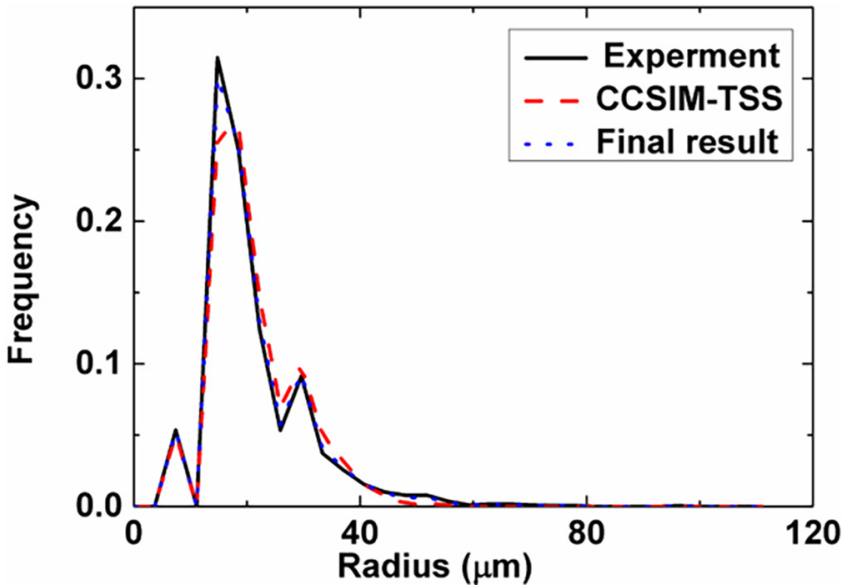

Fig. 9. Curves of the pore size distribution of the final reconstructed result, the realization of CCSIM-TSS and the experiment for carbonate sample C.

properties of the real samples. Furthermore, the pore network before and after optimization are both plotted together and compared with each other, as shown in Fig. 10. It can be seen from the figure that the optimization method based on MOSA cannot change the main connective path of the pore structure, however, it can change the digital core with the local micro-adjustment.

Finally, the absolute permeability of the final result obtained by the

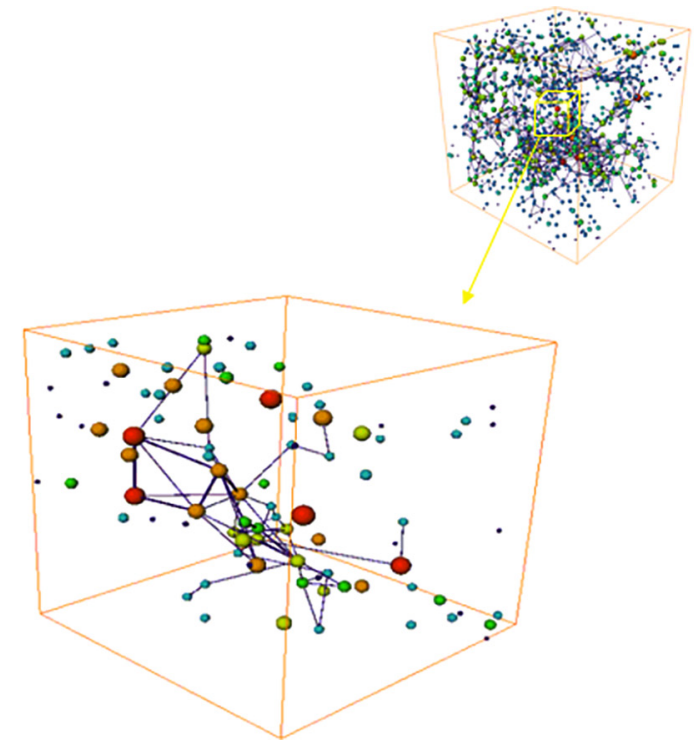

Table 1

Comparison of the permeability obtained from the reconstructed models for carbonate sample $\mathrm{C}$ and the experiment.

\begin{tabular}{lllll}
\hline \multirow{2}{*}{ Permeability $(\mathrm{mD})$} & Experiment & \multicolumn{3}{l}{ number of iterations } \\
\cline { 3 - 5 } & & 1 & 100 & 238 \\
\hline $\mathrm{K}_{\mathrm{x}}$ & 198 & 140 & 160 & 204 \\
$\mathrm{~K}_{\mathrm{y}}$ & 323 & 201 & 263 & 308 \\
$\mathrm{~K}_{\mathrm{z}}$ & 248 & 117 & 204 & 209 \\
\hline
\end{tabular}

optimization method are investigated. For sake of comparison, the absolute permeability of the realizations obtained by different number of iterations and the real rock sample are listed in Table 1. It can be seen that as the number of iterations increases, the absolute permeability of the realization is approaching to that of the real rock sample and the realization becomes more accurate.

\subsection{Tight sandstone samples}

To further test the described methodology, a tight sandstone sample in China, referred to as T, was selected. Compared to carbonate rock C, this sample represents a more complex case with micro-nanopores. The porosity of the tight sandstone sample is $4.2 \%$. Pulse decay permeability was performed at effective stress of $30.0 \mathrm{MPa}$ at the temperature of $38{ }^{\circ} \mathrm{C}$ that yielded an absolute permeability of $1.25 \mathrm{mD}$ based on the Klinkenberg expression (Bhandari et al., 2015). Thus, a 3D realization

Fig. 10. The pore network model of the final reconstructed result (a) and the realization of CCSIM-TSS (b) for carbonate sample C. 

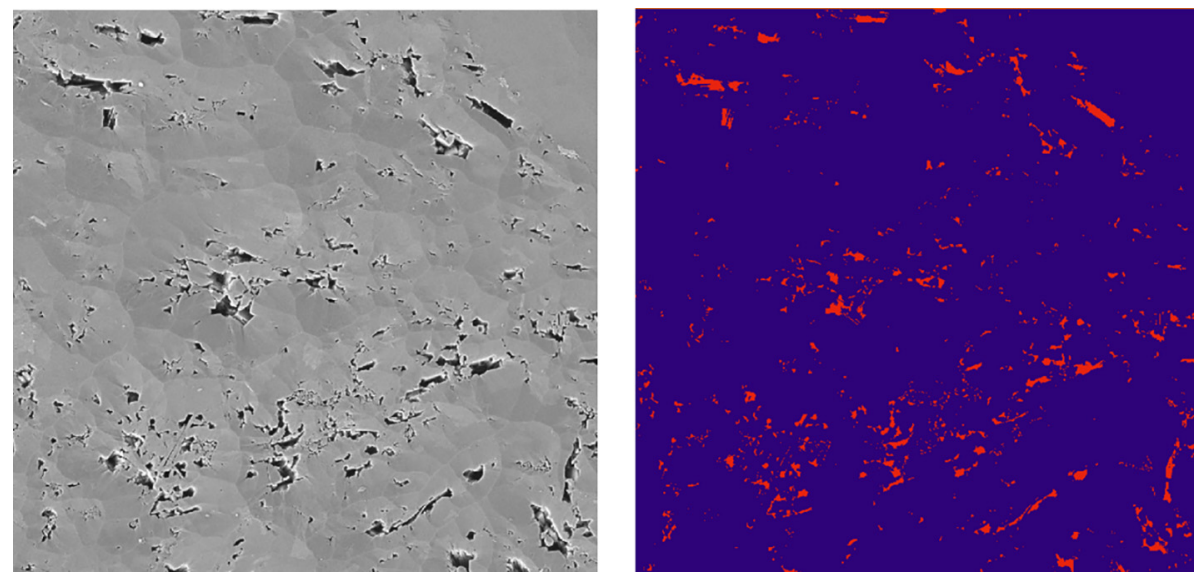

Fig. 11. The extracted 2D representative image for the tight sandstone sample.

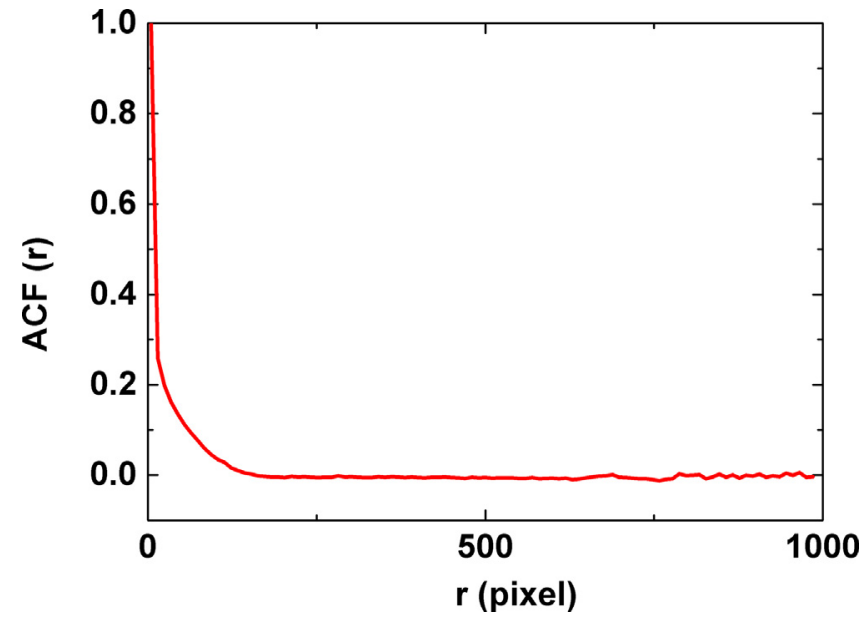

Fig. 12. The curve of the two-point connectivity probability function for the tight sandstone sample.

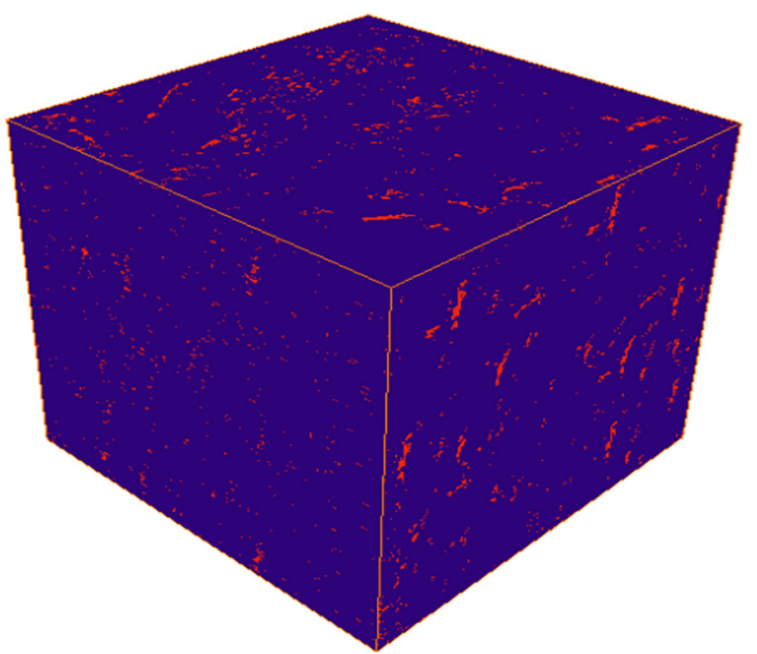

Fig. 13. 3D view of reconstructed model for the tight sandstones sample.

was generated using the CCSIM-TSS algorithm; see Fig. 1. Then, the optimization method was carried out to minimize the difference between the physical properties of the reconstructed 3D digital core and the realistic one.

In this paper, an SEM image of the tight sandstones sample, shown in Fig. 11, was used. The image consists of with $1000 \times 1000$ elements and a voxel size of $50 \mathrm{~nm}$. The image indicates a spatially stationary distribution of pores and the single DI was therefore used for different directions.

Also, based on the 2D image in Fig. 11, the two-point connectivity probability functions (ACF (r)) are extracted, as shown in Fig. 12. And it will be used as vertical constraint conditions in CCSIM-TSS for reconstructing $3 \mathrm{~d}$ models for the tight sandstone sample.

Based on the CCSIM-TSS method and the $2 \mathrm{~d}$ image, the realization for the tight sandstone sample is reconstructed, as shown in Fig. 13. Then the connectivity and the pore-size distribution of the produced realizations ware estimated. First, the two-point connectivity probability functions in the three directions are computed and then compared with that with the SEM image. Inspecting Fig. 14, one can find that there is a difference of the two-point connectivity probability functions between the reconstructed model and the SEM image when $r<200$ and $r \geqslant 900$. Next, the error, the difference between the computed pore size distribution and the experimental value (mercury intrusion porosimetry), was calculated. It can be seen from Fig. 15 that the difference of the pore-size distribution for the experiment and the realization is relatively big, especially for radius bigger than $200 \mu \mathrm{m}$. Therefore, similar to carbonate rock, it can be also obtained that realizations generated by the CCSIM-TSS method is not capable of reproducing accurately the connectivity and the pore-size distribution of the actual tight sandstone sample. Thus in the following we will use the optimization method to adjust the realization to minimize the difference between the realization and the actual sample.

The final digital core for the tight sandstone sample $\mathrm{T}$ is obtained after 1365 iterations. For the sake of comparison, the two-point connectivity function in three directions of the final model obtained by the optimization method, the CCSIM-TSS method and the SEM image are plotted in Fig. 16. It can be seen clearly that the connectivity of the final digital core have been improved greatly and the difference of the twopoint connectivity function between the final digital core obtained by the optimization method and the SEM image is very small. Also, the pore size distribution of the final digital core are examined. The comparison is shown in Fig. 17. Inspecting the figure, we can obtain that the final digital core reproduces the pore-throat size distribution of the experiment. The porosity associated with each of the original sample and generated realization was also computed to be $4.20 \%$ and $4.38 \%$, accordingly.

Finally, the permeability of the final result for tight sandstone sample $\mathrm{T}$ obtained by the optimization method are examined. For sake of comparison, the permeability of the realizations obtained by different number of iterations and the experiment are listed in Table 2. The absolute permeability changes from $035 \mathrm{mD}$ to $0.92 \mathrm{mD}$ to $1.13 \mathrm{mD}$ after 1, 600, and 1365 iterations, respectively. It should be noted that the permeability for the $3 \mathrm{D}$ samples were calculated based on the $\mathrm{AB}$ 

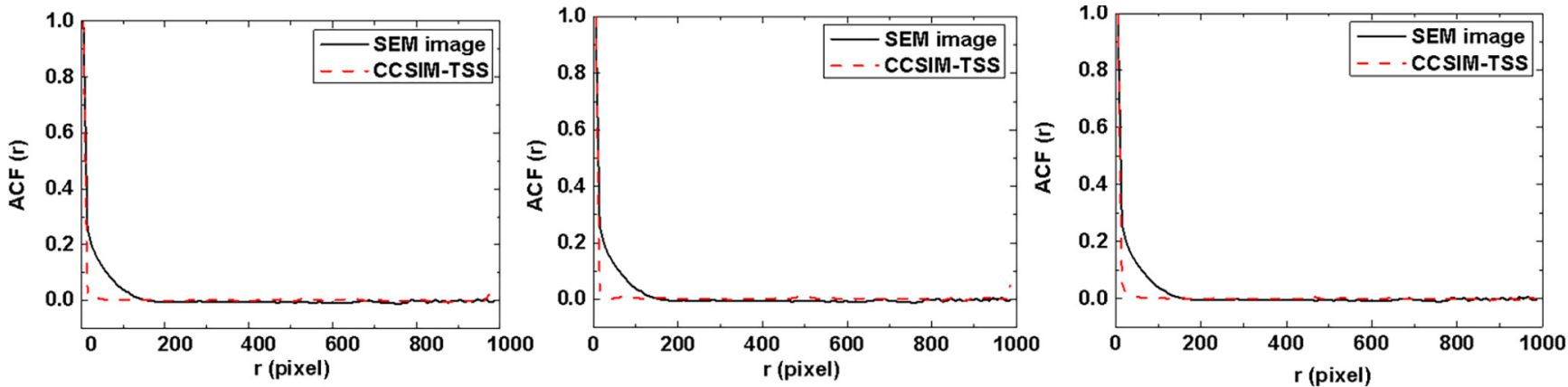

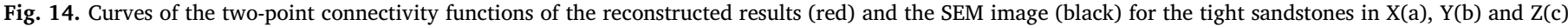
direction. (For interpretation of the references to colour in this figure legend, the reader is referred to the web version of this article.)

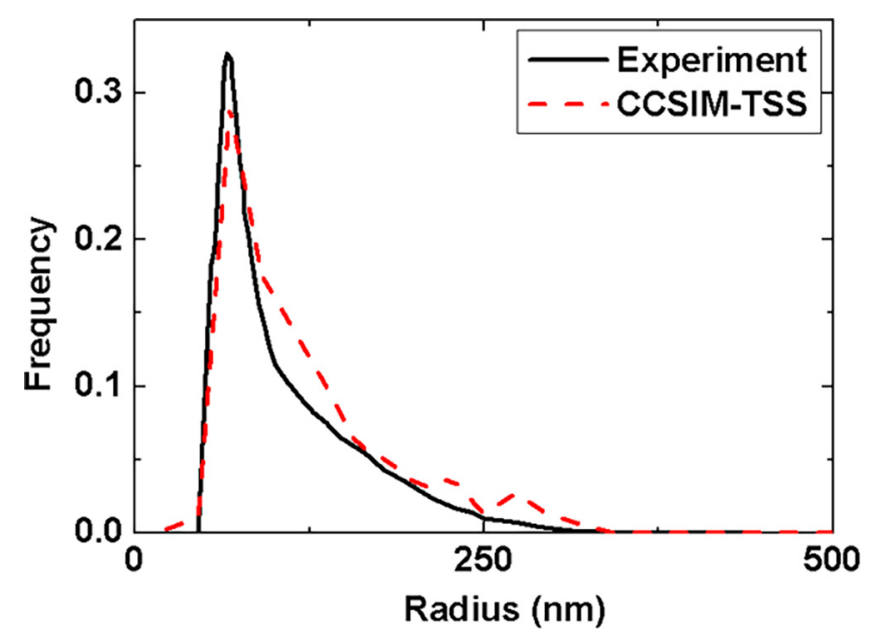

Fig. 15. Curves of the pore size distribution of the reconstructed results (red) and the experiment (black) for the tight sandstones. (For interpretation of the references to colour in this figure legend, the reader is referred to the web version of this article.)

algorithm, which result in a very fast evaluation. Also, it can be deduced that in the tight sandstones sample $\mathrm{T}$, the pores with radius around $100 \mathrm{~nm}$ have a large proportion and the connectivity of these pores have a strong impact on the intrinsic permeability of the tight sandstones.

\subsection{Analysis and discussion}

We have examined the hybrid method proposed in this paper for carbonate rock (C) and tight sandstones sample (T), and in this section we will further investigate the difference of performance of the hybrid algorithm on different heterogeneous porous media. For the sake of contrast, another carbonate rock (Cs), tight sandstones sample (Ts) and

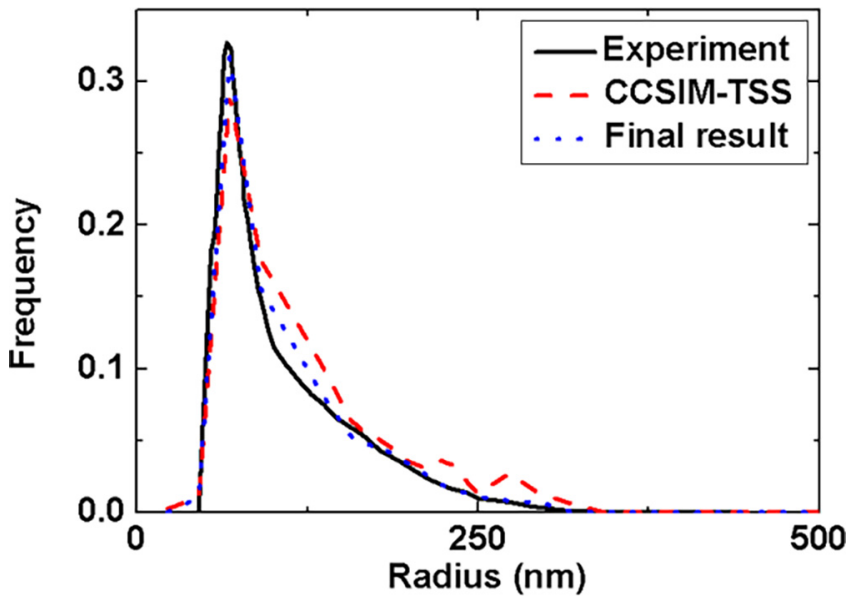

Fig. 17. Comparison of the pore size distribution of the final result obtained by the optimization method, the CCSIM-TSS method and the experiment.

Table 2

Comparison of the permeability obtained from the reconstructed models for the tight sandstone Sample (T) and the experiment.

\begin{tabular}{lllll}
\hline Permeability $(\mathrm{mD})$ & Experiment & \multicolumn{4}{l}{ number of iterations } \\
& & 1 & 600 & 1365 \\
$\mathrm{~K}$ & 1.25 & 0.54 & 0.82 & 1.03 \\
\hline
\end{tabular}

the homogeneous sandstones (S and Ss) are also reconstructed by the hybrid method here.

To examine the performance of the hybrid algorithm on different heterogeneous porous media more carefully, we calculate the pore size distribution and the relative errors of the models generated by CCSIMTSS method and the hybrid method for each sample in Fig. 18 and Table 3. Moreover, to investigate the heterogeneity of pore spaces of
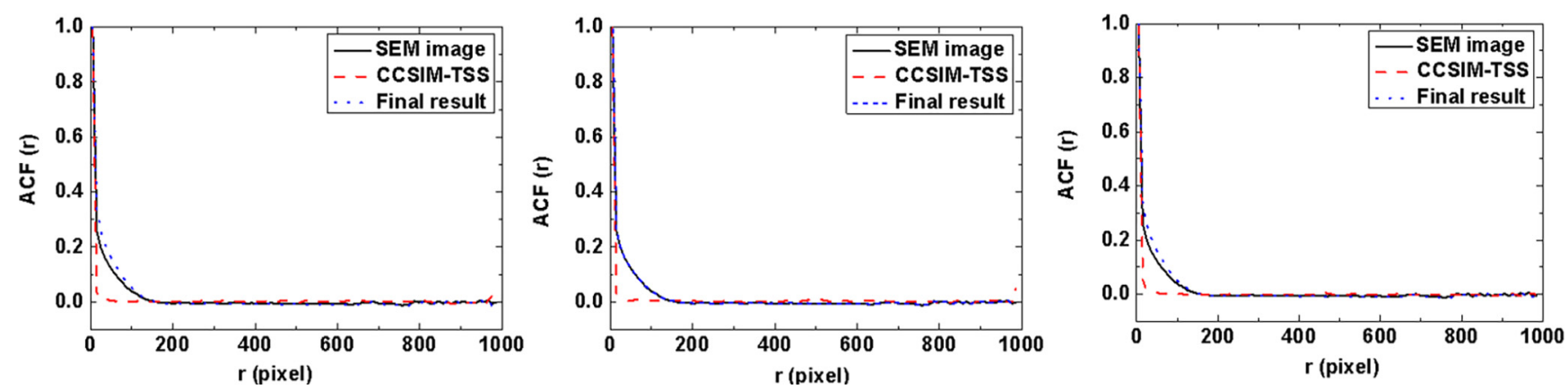

Fig. 16. Comparison of the two-point connectivity functions of the final model obtained by the optimization method, the CCSIM-TSS method and the SEM image in $X$ (a), $\mathrm{Y}(\mathrm{b})$ and $\mathrm{Z}(\mathrm{c})$ direction. 

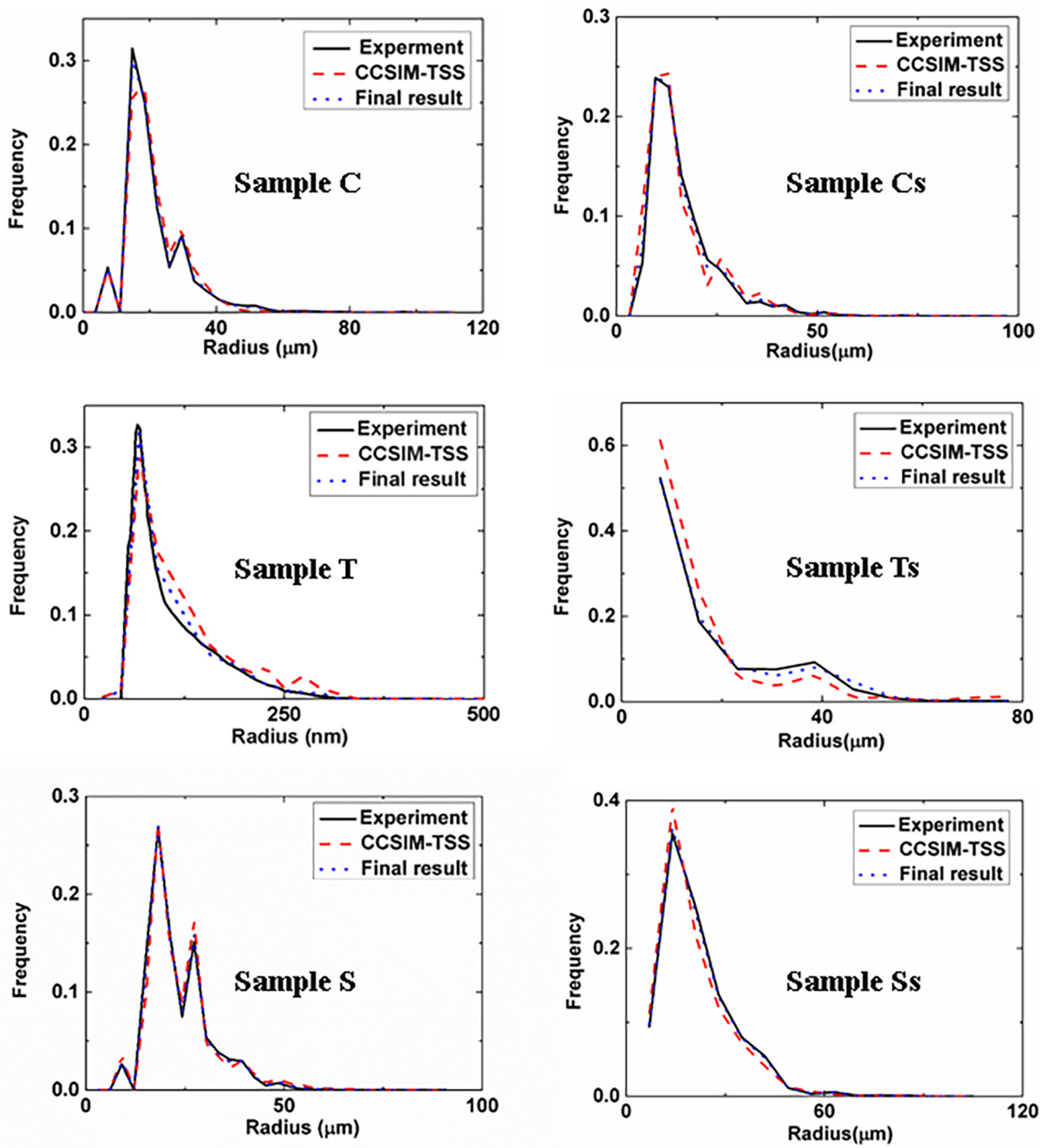

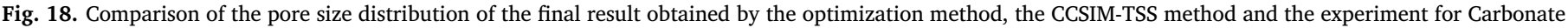
rock (C and $\mathrm{Cs}$ ), tight sandstone Sample ( $\mathrm{T}$ anns $\mathrm{Ts}$ ) and the sandstones (S and Ss).

the six rock samples quantitatively, Table 3 also compares the mean, variance and the ration between variance and mean (signed by V) of the pore size distribution of the six rock samples. A striking feature of Table 3 is that the tight sandstone sample Ts have the biggest value of $\mathrm{V}$, indicating that tight sandstone sample Ts have widest size range of pores and its pore space is more heterogeneous than the other rock samples. We can also deduce from Table 3 that the pore space of sandstone Ss is the most homogeneous one and the pore space of the two carbonate rocks are more homogeneous than the two tight sandstone samples.

Table 3 also illustrates the relative errors of the pore-size distribution and the permeability for the CCSIM-TSS and hybrid method for each sample. Inspecting Fig. 18 and Table 3, it can be found that the pore-size distribution and the permeability of the models generated by the CCSIM-TSS method for the homogeneous sandstones S and Ss have a relatively small difference with experiment, and have smaller relative errors than the other four rock samples. Then using the optimization method the relative errors for sample $\mathrm{S}$ and Ss decreases rapidly after several iterations. However, for carbonate rocks and tight sandstones samples, the relative errors of the pore-size distribution and the permeability of the models generated by the CCSIM-TSS method are much bigger than that for sample $S$ and Ss, while tight sandstones samples have the largest relative errors. The relative errors of the pore-size distribution (and the permeability) of the realizations for sample C, Cs, $\mathrm{T}$ and $\mathrm{Ts}$ decrease to $3.23 \%, 6.09 \%, 10.64 \%$ and $7.34 \%(14.5 \%$, $16.02 \%, 17.6 \%$ and $21.62 \%$ ) after 238, 520, 1365 and 1687 iterations, respectively. The numbers of iterations for tight sandstones samples are much bigger than that of carbonate rocks. This is because that the tight sandstone samples, whose pore structures have the widest range of length scales, are more heterogeneous than carbonate rocks. Thus we 
Table 3

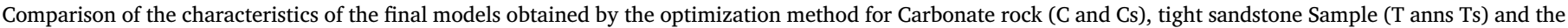
sandstones (S and Ss).

\begin{tabular}{|c|c|c|c|c|c|c|c|}
\hline \multirow[t]{2}{*}{ Samples } & & \multicolumn{2}{|c|}{ Carbonate rock } & \multicolumn{2}{|c|}{ Tight sandstones } & \multicolumn{2}{|c|}{ Sandstones } \\
\hline & & $\mathrm{C}$ & Cs & $\mathrm{T}$ & Ts & $\mathrm{S}$ & Ss \\
\hline \multirow[t]{3}{*}{ Pore size } & Mean $(\mu \mathrm{m})$ & 21.50 & 7.43 & 0.096 & 9.26 & 21.70 & 17.11 \\
\hline & Variance $(\mu \mathrm{m})$ & 9.44 & 13.40 & 0.059 & 7.04 & 8.03 & 5.73 \\
\hline & Variance/mean & 0.44 & 0.55 & 0.61 & 0.76 & 0.37 & 0.33 \\
\hline \multirow[t]{2}{*}{ CCSIM-TSS Relative Errors } & Pore-size distribution & $12.7 \%$ & $18.27 \%$ & $28.10 \%$ & $28.81 \%$ & $14.33 \%$ & $14.18 \%$ \\
\hline & Permeability & $40.41 \%$ & $46.31 \%$ & $56.80 \%$ & $66.31 \%$ & $11.01 \%$ & $12.91 \%$ \\
\hline Iteration Numbers & & 238 & 520 & 1365 & 1687 & 100 & 100 \\
\hline \multirow[t]{2}{*}{ Final results Relative Errors } & Pore-size distribution & $3.23 \%$ & $6.09 \%$ & $10.64 \%$ & $7.34 \%$ & $1.34 \%$ & $2.82 \%$ \\
\hline & Permeability & $14.50 \%$ & $16.02 \%$ & $17.60 \%$ & $21.62 \%$ & $5.19 \%$ & $6.71 \%$ \\
\hline
\end{tabular}

can conclude that the hybrid method proposed in this paper is more suitable for heterogeneous porous media and the properties of the realizations reconstructed by this method have a very good agreement with that of actual cores. As the heterogeneity of the pore space increases, the number of iterations of the hybrid method becomes larger in order to obtain the high quality realizations.

As is well known, stochastic methods must be conditioned further to produce certain important properties. One can use more experimental data (such as electrical conductivity) as the objective functions to reduce the uncertainty and produce more realistic realizations.

\section{Summary}

In this paper, a hybrid method based on CCSIM-TSS method and the optimization method is proposed to reconstruct the digital core model of heterogeneous pore structure of porous media. After analysis of digital rocks, some important conclusions are obtained as follows:

(1) In the hybrid method, the two-point connectivity function, the multiple-point connectivity function, the pore size distribution and the permeability are used as the objective functions. Moreover, the hybrid method proposes the generation solutions of the new system by exchanging the objective points and boundary points of pore and matrix based on the two algorithms.

(2) Quantitative comparison is made by computing the two-point connectivity function, permeability and pore-size distributions for the realizations generated by the hybrid method, as well as the original samples. The hybrid algorithm was demonstrated to be able to produce higher quality realizations than CCSIM-TSS method for heterogeneous porous media.

(3) The method generates realization of tight sandstones that match the measured permeability and the pore size distribution based on 2D SEM image without using full 3D imaging. Clearly, the reconstructed digital core improves our knowledge of the pore-network connectivity of tight sandstones. The method may also be used with various types of experimental data, such as the permeability, electrical conductivity. Clearly, more experimental data reduce the more realistic realizations.

\section{Acknowledgements}

This work is supported by the National Program on Key Basic Research Project (973 Program, Grant No. 2014CB239004), the Strategic Priority Research Program of the Chinese Academy of Sciences (grant No. XDA14010304), the Major National Science and Technology Special Program of China (Grant No. 2017ZX05037-001), the National Natural Science Foundation of China (Grant No. 41690132) and the Strategic Priority Research Program of the Chinese Academy of Sciences (Grant No. XDB10020302).

\section{References}

Bai, B., Lgmati, M., Hang, H., Wei, M., 2013. Rock characterization of Fayetteville shale gas plays. Fuel 105 (1), 645-652.

Bhandari, A.R., Flemings, P.B., Polito, P.J., Cronin, M.B., Bryant, S.L., 2015. Anisotropy and tress dependence of permeability in the Barnett shale. Transp. Porous Media 108 (2), 393-411.

Biswal, B., Manwart, C., Hilfer, R., et al., 1999. Quantitative analysis of experimental and synthetic microstructures for sedimentary rock. Physica A 273 (3), 452-475.

Biswal, B., Øren, P.E., Held, R.J., et al., 2007. Stochastic multiscale model for carbonate rocks. Phys. Rev. E 75 (6), 061303.

Bryant, S., Blunt, M., 1992. Prediction of relative permeability in simple porous media. Phys. Rev. A 46 (4), 2004-2011.

Chen, X., Zhou, Y., 2017. Applications of digital core analysis and hydraulic flow units in petrophysical characterization. Adv. Geo-energ. Res 1 (1), 18-30.

Coelho, D., Thovert, J.F., Adler, P.M., 1997. Geometrical and transport properties of random packings of spheres and aspherical particles. Phys. Rev. E 55 (2), 1959-1978.

Curtis, M.E., Sondergeld, C.H., Ambrose, R.J., Rai, C.S., 2012. Microstructural investigation of gas shales in two and three dimensions using nanometer-scale resolution imaging. AAPG Bull. 96 (4), 665-677.

Gao, M.L., He, X.H., Teng, Q.Z., Zuo, C., Chen, D.D., 2015. Reconstruction of three-dimensional porous media from a single two-dimensional image using three-step sampling. Phys. Rev. E 91, 013308.

Hajizadeh, A., Farhadpour, Z., 2012. An algorithm for 3D pore space reconstruction from a 2D image using sequential simulation and gradual deformation with the probability perturbation sampler. Transp. Porous. Med. 94 (3), 859-881.

Hajizadeh, A., Safekordi, A., Farhadpour, Z., 2011. A multiple-point statistics algorithm for 3D pore space reconstruction from 2D images. Adv. Water Resour. 34 (10), 1256-1267.

Ji, L.L., Lin, M., Jiang, W.B., et al., 2018. An improved method for reconstructing the digital core model of heterogeneous porous media. Transp. Porous Media. http://dx.doi.org/10. 1007/s11242-017-0970-5.

Kelly, S., El-Sobky, H., Torres-Verdin, C., Balhoff, M.T., 2016. Assessing the utility of FIB-SEM images for shale digital rock physics. Adv. Water Resour. 95, 302-316.

Krishnan, S., Journel, A.G., 2003. Spatial connectivity: from variograms to multiple-point measures. Math. Geol. 5 (8), 915-925.

Liu, Z.H., Yang, Y.F., Yao, J., Qi, Z., Ma, J.S., Qian, Q., 2017. Pore-scale remaining oil distribution under different pore volume water injection based on CT technology. Adv. Geoenerg. Res 1 (3), 171-181.

$\emptyset$ ren, P.E., Bakke, S., 2002. Process based reconstruction of sandstones and prediction of transport properties. Transp. Porous. Med. 46 (2-3), 311-343.

Payne, S.S., Wild, P., Lubbe, R., 2010. An integrated solution to rock physics modeling in fractured carbonate reservoirs. In: Annual International Meeting, 80th, Society of Exploration Geophysicists. vol. 1. Society of Exploration Geophysicists, Tulsa, Okla, USA, pp. 358-362.

Pyrcz, M.J., Deutsch, C.V., 2014. Geostatistical Reservoir Modeling. Oxford University Press.

Strebelle, S., 2002. Conditional simulation of complex geological structures using multiplepoint geostatistics. Math. Geol. 34, 1-22.

Strebelle, S., Cavelius, C., 2014. Solving speed and memory issues in multiple-point statistics simulation program SNESIM. Math. Geol. 46 (2), 171-186.

Tahmasebi, P., Hezarkhani, A., Sahimi, M., 2012. Multiple-point geostatistical modeling based on the crosscorrelation functions. Comput. Geosci. 16 (3), 779-797.

Tahmasebi, P., Javadpour, F., Sahimi, M., 2015. Three-dimensional stochastic characterization of shale SEM images. Transp. Porous. Med. 110, 521-531.

Tahmasebi, P., Javadpour, F., Sahimi, M., Piri, M., 2016. Multiscale study for stochastic characterization of shale samples. Adv. Water Resour. 89, 91-103.

Tahmasebi, P., Sahimi, M., 2016. Enhancing multiple-point geostatistical modeling: 1. Graph theory and pattern adjustment. Water Resour. Res. 52 (3), 2074-2098.

Weger, R.J., Eberli, G.P., Baechle, G.T., et al., 2009. Quantification of pore structure and its effect on sonic velocity and permeability in carbonates. AAPG Bull. 93 (10), 1297-1317.

Wu, K., Nunan, N., Crawford, J.W., et al., 2004. An efficient Markov chain model for the simulation of heterogeneous soil structure. Soil Sci. Soc. Am. 68, 346-351.

Yi, Z.X., Lin, M., Jiang, W.B., et al., 2017. Pore network extraction from pore space images of various porous media systems. Water Resour. Res. 53 (4), 3424-3445.

Zhou, S., Yan, G., Xue, H., Guo, W., Li, X., 2016. 2D and 3D nanopore characterization of gas shale in Longmaxi formation based on FIBSEM. Mar. Pet. Geol. 73, 174-180. 